\title{
THE HOHEISEL PHENOMENON FOR GENERALIZED DIRICHLET SERIES
}

\author{
CARLOS JULIO MORENO
}

\begin{abstract}
Hoheisel's proof that the difference between two consecutive primes is of smaller order of magnitude than either prime depends on Littlewood's estimate for the zero-free region of the Riemann zeta function and a density estimate for the number of zeros in certain rectangles in the critical strip. In this note we derive Hoheisel's result without appealing to Littlewood's theorem, thus enlarging the range of applicability of Hoheisel's argument to a more general class of Dirichlet series. Applications of the results to number theory are given.
\end{abstract}

1. Introduction. Hoheisel proved in [3] that if $p_{n}$ is the $n$th prime, then

$$
p_{n+1}-p_{n} \ll p_{n}^{\theta},
$$

where $\theta<1$. All known proofs of this result and its improved versions have been made to depend on two propositions concerning the distribution of zeros of the Riemann zeta function

$$
\zeta(s)=\sum_{n=1}^{\infty} n^{-s}, \quad s=\sigma+i t,
$$

namely, Littlewood's theorem (or the stronger Tchudakoff theorem) which asserts that $\zeta(s) \neq 0$ in the region $\sigma \geqq 1-(A \log \log t) / \log t$ and a density estimate of the type

$$
N(\sigma, T) \ll T^{c(1-\sigma)}(\log T)^{b}, \quad c>0, b \geqq 0,
$$

uniformly for $\frac{1}{2} \leqq \sigma \leqq 1$ as $T \rightarrow \infty$. Here $N(\sigma, T)$ is the counting function defined by $N(\sigma, T)=\#$ of zeros $\rho=\beta+i \gamma$ of $\zeta(s)$ with $\beta \geqq \gamma$ and $|\gamma| \leqq T$. (See for example Ingham [4]. For an interesting discussion of the literature see Chandrasekharan [1] and Montgomery [5].) The aim of this note is to derive a result of the type (1) without the assumption of Littlewood's theorem. This is achieved at the expense of having to establish a density

Received by the editors May 9, 1972.

AMS (MOS) subject classifications (1970). Primary 10D05, 10H25; Secondary $10 \mathrm{H} 10$.

Key words and phrases. Hoheisel phenomenon, Dirichlet series, Ramanujan functions $\tau(n)$, Littlewood theorem. 
estimate (2) without the logarithm factors as has already been done by Gallagher [2] and others.

2. Statement of results. For practical purposes we treat a more general situation. Let

$$
\varphi(s)=\sum_{n=1}^{\infty} a_{n} n^{-s}, \quad s=\sigma+i t,
$$

be a Dirichlet series with nonnegative coefficients. Define the function $N_{\varphi}(\sigma, T)=\#$ of zeros $\rho=\beta+i \gamma$ of $\varphi(s)$ with $\beta \geqq \sigma$ and $|\gamma| \leqq T$. We say that $\varphi(s)$ has the Hoheisel Property if the following four properties hold:

$$
\psi(x)=\sum_{p \leqq x} a_{p} \log p=x-\sum_{|\gamma| \leqq T} x^{\rho} / \rho+O\left(x(\log T x)^{2} / T\right),
$$

where the first sum is over primes and the second is over the zeros $\rho=$ $\beta+i \gamma$ of $\varphi(s)$ with $|\gamma| \leqq T \leqq x^{1 / 2}$ and $\beta \geqq 0$.

$$
\begin{gathered}
\varphi(s) \neq 0 \text { in the region } \sigma \geqq 1-a / \log (2+|t|), \quad a>0 . \\
N_{\varphi}(\sigma, T) \ll T^{c(1-\sigma)}, \quad c>0,
\end{gathered}
$$

uniformly for $\frac{1}{2} \leqq \sigma \leqq 1$, as $T \rightarrow \infty$.

$$
N_{\varphi}(0, T) \ll T \log T
$$

The result we prove is the following.

MAIN Theorem. If the Dirichlet series $\varphi(s)=\sum_{n=1}^{\infty} a_{n} n^{-s}$ has the Hoheisel Property, then a positive constant $v<1$ can be found such that

$$
\sum_{x \leqq p \leqq x+h} a_{p} \log p \geqq c_{0} h, \quad c_{0}>0,
$$

for any $h=x^{\theta}$ with $\theta>v$.

2.1. Remarks. The best known example of a Dirichlet series with the Hoheisel Property is the Dedekind zeta function of a cyclotomic field. In this case the arithmetical equivalent of the Hoheisel Property is the result

$$
\sum_{x \leqq p \leqq x+h ; p \equiv a(\bmod m)} \log p=(h / \varphi(m))(1+o(1)),
$$

which holds for any $h \geqq x^{\theta}$ and $x \geqq x_{0}(m)$; $\theta$ is a positive real number satisfying $\theta<1$, possibly $\theta<\frac{1}{2}+\varepsilon$. The result in (4) can also be given the following algebraic interpretation: let $g$ be an element of the Galois group $G_{m}$ of the cyclotomic extension $Q\left(e^{2 \pi i / m}\right)$ obtained by adjoining the $m$ th roots of 1 to the rationals; (4) now implies that any interval of the 
type $[x, x+h]$ contains at least one rational prime whose Frobenius element in $G_{m}$ is the given $g$.

2.2. The algebraic interpretation of the Hoheisel phenomenon given above has been generalized by the author to algebraic number fields whenever the formalism of the Artin $L$-functions is possible. The main result obtained in this direction is a sharpening of Tchebotarev's density theorem to the effect that given any conjugacy class $C$ in the Galois group $G(K / Q)$ of a normal extension $K$ of the rationals, a rational prime $p$ can always be found in the interval $\left[x, x+x^{\theta}\right]$ ( $x$ sufficiently large and $\theta=$ $\left.\theta_{0}(K)<1\right)$, such that the Frobenius element $\sigma_{p} \in G(K / Q)$ generated by $p$ is in the given conjugacy class. The proof of this result uses the Main Theorem of this paper. The technical difficulties consist in establishing that the Artin $L$-functions attached to $K$ have the Hoheisel Property. The details will appear elsewhere.

2.3. The investigations presented in this paper were suggested to the author by the classical problem of whether the Ramanujan function $\tau(n)$ ever vanishes. The present methods are not strong enough to solve completely this problem; nevertheless, using the Hoheisel phenomenon, we can now give some information about intervals which contain primes where $\tau(p)$ does not vanish. Recall that the Ramanujan function $\tau(n)$ is defined by formally expanding the infinite product

$$
q \prod_{n=1}^{\infty}\left(1-q^{n}\right)^{24}=\sum_{n=1}^{\infty} \tau(n) q^{n}
$$

The relevant Dirichlet series that one must consider in this case is

$$
\varphi(s)=\sum_{n=1}^{\infty} \tau^{2}(n) n^{-11-s}
$$

The methods developed by the author for proving prime number theorems for the coefficients of modular forms lead to the following explicit formula

$$
\sum_{p \leqq x} \tau(p)^{2} p^{-11} \log p=x-\sum_{|\gamma| \leqq T} x^{\rho} / \rho+O\left(x(\log T x)^{2} / T\right),
$$

where the second sum is over the zeros $\rho=\beta+i \gamma$ of $\varphi(s)$ with $|\gamma| \leqq T \leqq x^{1 / 2}$ and $\beta \geqq 0$. In fact we have shown that the Dirichlet series $\varphi(s)$ has the Hoheisel Property. If we now apply the Main Theorem of this paper to (5) we obtain that

$$
\sum_{x \leqq p \leqq x+x^{\theta}} \tau(p)^{2} p^{-11} \log p \gg x^{\theta},
$$

for some $\theta<1$ and all $x \geqq x_{0}$. If $|\tau(p)| \leqq 2 p^{11 / 2}$, then the inequality (6) leads 
to the fact that

$$
\#\left\{p \mid x \leqq p \leqq x+x^{\theta} \text { and } \tau(p) \neq 0\right\} \gg x^{\theta} / \log x .
$$

Further details concerning the method of prime number theorems for the coefficients of modular forms and the proof that the Dirichlet series $\varphi(s)$ in (5) has the Hoheisel Property can be found in [6] and [7].

3. Proof of Main Theorem. From (A) we have

$$
\{\psi(x+h)-\psi(x)\} / h=1-\sum_{|\gamma| \leqq T}\left\{(x+h)^{\rho}-x^{\rho}\right\} / h \rho+O\left(x(\log T x)^{2} / h T\right) .
$$

Observe that $\left\{(x+h)^{\rho}-x^{\rho}\right\} / h \rho=(1 / h) \int_{x}^{x+h} y^{\rho-1} d y \ll x^{\beta-1}$. Hence

(7) $\{\psi(x+h)-\psi(x)\} / h=1+O\left(\sum_{|\gamma| \leqq T} x^{\beta-1}\right)+O\left(x(\log T x)^{2} / h T\right)$.

To estimate the sum in (7) we use the method of Stieltjes integration as follows

where

$$
\begin{aligned}
\sum_{|\gamma| \leqq T}\left(x^{\beta-1}-x^{-1}\right) & =\sum_{|\gamma| \leqq T} \int_{0}^{\beta} x^{\sigma-1} \log x d \sigma \\
& =\sum_{j=1}^{\lambda(T)} \sum_{\beta ; \rho=\beta+i \gamma_{j}} \int_{0}^{\beta} x^{\sigma-1} \log x d \sigma \\
& =\sum_{j=1}^{\lambda(T)} \sum_{\beta ; \rho=\beta+i \gamma_{j}} \int_{0}^{1} \delta_{\beta}(\sigma) x^{\sigma-1} \log x d \sigma,
\end{aligned}
$$

$$
\begin{aligned}
\delta_{\beta}(\sigma)=1 & \text { if } \sigma \leqq \beta, \\
=0 & \text { if } \sigma>\beta .
\end{aligned}
$$

Interchanging the order of summation and integration in (8) we obtain

where

$$
=\int_{0}^{1} f(\sigma) x^{\sigma-1} \log x d \sigma
$$

$$
f(\sigma)=\sum_{j=1}^{\lambda(T)} \sum_{\beta ; \rho=\beta+i \gamma_{j}} \delta_{\beta}(\sigma)
$$

is our former $N_{\varphi}(\sigma, T)$. Using (C) and (D) and observing that $N_{\varphi}(\sigma, T)=0$, for $\sigma \geqq 1-A / \log T=1-\eta(T)$,

$$
\begin{aligned}
\sum_{|\gamma| \leqq T} x^{\beta-1} & =N_{\varphi}(0, T) / x+O\left(\int_{0}^{1-\eta(T)} N_{\varphi}(\sigma, T) x^{\sigma-1} \log x d \sigma\right) \\
& =O((T \log T) / x)+O\left(\int_{0}^{1-\eta(T)}\left(T^{c} / x\right)^{1-\sigma} \log x d \sigma\right) .
\end{aligned}
$$


Let $T=x^{\alpha}$ where $\alpha$ is a small positive number to be chosen suitably later on. The integral in (9) now becomes

$$
\begin{aligned}
& \int_{0}^{1-\eta\left(x^{\alpha}\right)} x^{(\alpha c-1)(1-\sigma)} \log x d \sigma \\
& =\left(\exp \left[-(1-\alpha c) \eta\left(x^{\alpha}\right) \log x\right]-\exp [-(1-\alpha c) \log x]\right) /(1-\alpha c) \\
& =(\exp [-(1-\alpha c) A / \alpha]-\exp [-(1-\alpha c) \log x]) /(1-\alpha c) .
\end{aligned}
$$

The idea of the proof is to choose $\alpha$ so small that the right hand side of (10) dominates the constant in the $O$-term in (9). In fact we can choose an $\alpha$ such that

$$
\left|\sum_{|\gamma| \leqq T} x^{\beta-1}\right| \leqq \frac{1}{4}, \quad x \geqq x_{0} .
$$

Therefore from (7) and (11) we get

$$
\{\psi(x+h)-\psi(x)\} / h \geqq \frac{3}{4}-K x^{1-\alpha}(\log x)^{2} / h,
$$

where $K$ is an absolute constant. Clearly the left hand side of (12) will be larger than $\frac{1}{2}$ if $\left|K x^{1-\alpha}(\log x)^{2} / h\right| \leqq \frac{1}{4}$ which will certainly occur if $h \geqq 4 K x^{1-\alpha}(\log x)^{2}$. This completes the proof of the Main Theorem.

\section{REFERENCES}

1. K. Chandrasekharan, Arithmetical functions, Die Grundlehren der math. Wissenschaften, Band 167, Springer-Verlag, New York and Berlin, 1970. MR 43 \#3223.

2. P. X. Gallagher, A large sieve density estimate near $\sigma=1$, Invent. Math. 11 (1970), 329-339. MR 43 \#4775.

3. G. Hoheisel, Primzahl probleme in der Analysis, S.-B. Preuss. Akad. Wiss. 1930, $580-588$.

4. A. E. Ingham, On the difference between consecutive primes, Quart. J. Math. Oxford Ser. 8 (1937), 255-266.

5. H. L. Montgomery, Topics in multiplicative number theory, Lecture Notes in Math., vol. 227, Springer-Verlag, Berlin and New York, 1970.

6. C. J. Moreno, Prime number theorems for the coefficients of modular forms, Bull. Amer. Math. Soc. 78 (1972), 255-266.

7. - Prime number theorems for the coefficients of modular forms and a problem of G. H. Hardy (to appear).

8. - - A density estimate for the Ramanujan zeta function (to appear).

Center for Advanced Study, Graduate College, University of Illinois, URBANA, ILLINOIS 61801

Current address: Department of Mathematics, University of Illinois, Urbana, Illinois 61801 